doi: 10.18484/2305-0047.2019.3.354

\title{
В.В. БОЙКО ${ }^{1}$, Р.М. СМАЧИЛО ${ }^{1}$, Н.С. ЧЕРНЯЕВ ${ }^{2}$, И.И. ЯКОВЦОВА ${ }^{3}$, С.В. ДАНИЛЮК ${ }^{3}$, А.М. ТИЩЕНКО ${ }^{1}$, А.Л. ВЕСЕЛЫЙ ${ }^{1}$
}

\section{МУЛЬТИФОКАЛЬНАЯ АНГИОМИОЛИПОМА ПЕЧЕНИ И ЛЕГКИХ}

\author{
Институт общей и неотложной хирургии им. В.Т. Зайцева \\ Национальной Академии Медицинских Наук Украины ${ }^{1}$, \\ Харьковский национальный медицинский университет ${ }^{2}$, \\ Харьковская медицинская академия последипломного образования ${ }^{3}$, г. Харьков,
}

Украина

В статье представлен клинический случай хирургического лечения пациентки с редким онкологическим заболеванием - мультифокальной ангиомиолипомой печени и легких. Пациентка госпитализирована в клинику института, обследована, при спиральной компьютерной томографии органов брюшной полости и грудной клетки выявлены множественные образования легких билатерально, образования печени: в $\mathrm{Sg} 2$ и $\mathrm{Sg} 4-3$ образования до 2 см в диаметре, в $\mathrm{Sg} 1-$ крупное аналогичное образование $10 \times 8$ см, прилежащее к нижней полой вене. Выполнена дифференциальная диагностика с гепатоцеллюлярной карциномой при помощи определения онкомаркеров крови и трепан-биопсии опухоли хвостатой доли печени с последующим гистологическим исследованием. После подтверждения вероятного доброкачественного характера опухоли пациентка была оперирована. Интраоперационно в Sg2 и Sg4 печени выявлены образования до 2 см в диаметре; в $\mathrm{Sg} 1$ печени - опухоль размерами $9 \times 8 \times 10$ см, вплотную прилежащая к нижней полой вене, легкоранимая и обильно кровоточащая при контакте. Выполнена резекция Sg1 печени с опухолью левосторонним способом с использованием методики афферентной васкулярной эксклюзии печени, техники «clamp crush», электрохирургического сваривания. Послеоперационный период протекал гладко, без осложнений, пациентка выписана на 12-е послеоперационные сутки. Иммуногистохимическое исследование удаленного препарата подтвердило диагноз - ангиомиолипома печени.

Ключевые слова: ангиомиолипома, хвостатая доля печени, резекция печени, компьютерная томография, иммуногистохимическое исследование, электрохирургическая сварка

The article presents a clinical case of surgical treatment of a patient with a rare oncological disease multifocal angiomyolipoma of the liver and lungs. The patient had been hospitalized to the clinic, examined using spiral computed tomography of the abdominal organs and the chest. The following was revealed - bilateral multiple lung neoplasms, liver neoplasms: in $\mathrm{Sg} 2$ and $\mathrm{Sg} 4-3$ tumors up to $2 \mathrm{~cm}$ in diameter, in $\mathrm{Sg} 1-$ a large similar tumor 10x8cm adjacent to the inferior vena cava. Differential diagnostics with hepatocellular carcinoma was performed by using the determination of the blood tumor markers and trepan biopsy of the tumor of the caudate hepatic lobe, followed by histological examination. After confirming of probable benign nature of the tumor, the patient was operated on. Intraoperative findings were the following: in $\mathrm{Sg} 2$ and $\mathrm{Sg} 4$ of the liver - neoplasms up to $2 \mathrm{~cm}$ in diameter; in $\mathrm{Sg} 1$ of the liver - a neoplasm $9 \times 8 \times 10 \mathrm{~cm}$, closely adjacent to the inferior vena cava, easily vulnerable with bleeding after contact. Resection of $\mathrm{Sg} 1$ of the liver with a tumor was performed by the left-sided method using afferent vascular exclusion of the liver, clamp crush technique, and electrosurgical welding. The postoperative period was stable, without complications, the patient was discharged from hospital on the $12^{\text {th }}$ postoperative day. The immunohistochemical study of the removed tumor confirmed the diagnosis of liver angiomyolipoma.

Keywords: angiomyolipoma, caudate lobe of the liver, liver resection, computed tomography, immunohistochemistry, electrosurgical sealing

Novosti Khirurgii. 2019 May-Jun; Vol 27 (3): 354-359

The articles published under CC BY NC-ND license

Multifocal Angiomyolipoma of the Liver and Lungs

V.V. Boyko, R.M. Smachylo, M.S. Chernyayev, I.I. Yakovtsova, S.V. Danyliuk, O.M. Tyshchenko, A.L. Veselyi

\section{Введение}

Ангиомиолипома (АМЛ) - редкое доброкачественное новообразование, которое иногда имеет злокачественный потенциал [1, 2, 3, 4]. АМЛ обычно возникает у женщин и локализуется в почке, значительно реже - в печени и легких [5]. Микроскопически опухоль состоит из гетерогенной смеси гладких мышечных клеток, зрелой жировой ткани, толстостенных кровеносных сосудов с очагами экстрамедуллярного кроветворения, откуда и возникло название новообразования. Гистологически диагноз подтверждается иммуногистохимическим 
окрашиванием и выявлением ассоциированного с меланомой антигена (НМВ-45) [6, 7].

Возникновение АМЛ в печени и легких без одновременного развития их в почке является чрезвычайно редким явлением, и лишь несколько таких случаев было зарегистрировано в мировой литературе [8, 9]. В данной статье представлен клинический случай хирургического лечения пациентки с АМЛ печени и легких.

Цель. Представить собственный клинический случай мультифокальной ангиомиолипомы печени и проанализировать имеющиеся литературные данные об особенностях диагностики и лечения данного заболевания.

\section{Клинический случай}

13.03.2018 в клинику Института общей и неотложной хирургии им. В.Т. Зайцева поступила пациентка с жалобами на ноющие боли в правом подреберье, общую слабость. Считает себя больной в течение 1 года, когда впервые возникли вышеуказанные симптомы. Пациентка обследована. При спиральной компьютерной томографии органов грудной клетки и брюшной полости (КТ ОГК и ОБП) выявлены множественные образования легких билатерально, преимущественно в нижних долях, 3-18 мм в диаметре; образования печени: в $\mathrm{Sg} 1-10 \times 8$ см, прилежащее к нижней полой вене (НПВ); в Sg2 и $\mathrm{Sg} 4-3$ аналогичных образования до 2 см в диаметре (рис. 1).

Заключение КТ ОГК и ОБП: новообразования $\mathrm{Sg} 1, \mathrm{Sg} 2, \mathrm{Sg} 4$ печени и нижних долей обоих легких (Периваскулярные эпителиоидноклеточные опухоли (PEComa)? Гепатоцеллюлярный рак печени с метастатическим поражением легких?).

Пациентка была дообследована. Тест на альфа-фетопротеин негативный. Произведена трепан-биопсия опухоли Sg1 печени под У3- контролем. Гистологически структура образования более всего соответствовала ангиомиолипоме печени (рис. 2).

Накопленный опыт более 500 резекций печени позволил нам в качестве оптимального метода хирургического лечения выбрать резекционный - удаление новообразования S1 печени в пределах здоровых тканей.

Ход операции. Выполнена верхнесрединная лапаротомия. При ревизии в $\mathrm{Sg} 2$ и $\mathrm{Sg} 4$ печени - образования до 2 см в диаметре; в $\mathrm{Sg} 1$ печени - опухоль размерами $9 \times 8 \times 10 \mathrm{~cm}$, вплотную прилежащая к НПВ, мягкой консистенции, легкоранимая и обильно кровоточащая при контакте.

Выполнена резекция Sg1 печени с опухолью, при этом была использована методика афферентной васкулярной эксклюзии печени (Pringle-маневр) в режиме 5'-10'-15'. В ходе транссекции паренхимы нами была применена методика «clamp crush»: в плоскости резекции мягким зажимом разрушалась паренхима печени, при этом сосуды и желчные протоки диаметром более 1 мм оставались неповрежденными. Это давало возможность их дополнительно обрабатывать, при этом сосудистые структуры обрабатывали аппаратом электрохирургической сварки «Патонмед ЕКВ3-300», желчные протоки клипировали или лигировали. Все этапы вмешательства были выполнены с учетом принципов малообъемной инфузионной терапии при низких цифрах ЦВД (0-50 мм вод. ст.). Вид операционного поля после удаления опухоли S1 печени представлен на рис. 3 .

Новообразования легких, Sg2 и Sg4 печени было решено не удалять ввиду их множественности, небольших размеров и предположительного доброкачественного характера.

Удаленный препарат - резецированный участок Sg1 печени с новообразованием, окруженным нормальной паренхимой печени.

Рис. 1. Новообразования печени.

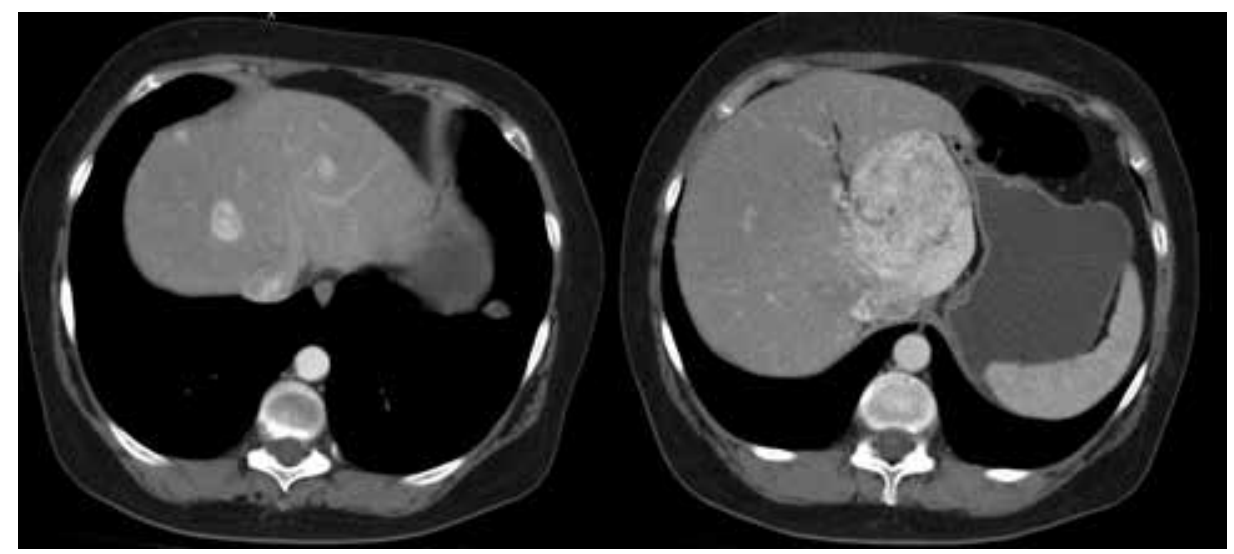


(c) В.В. Бойко с соавт. Ангиомиолипома печени и легких

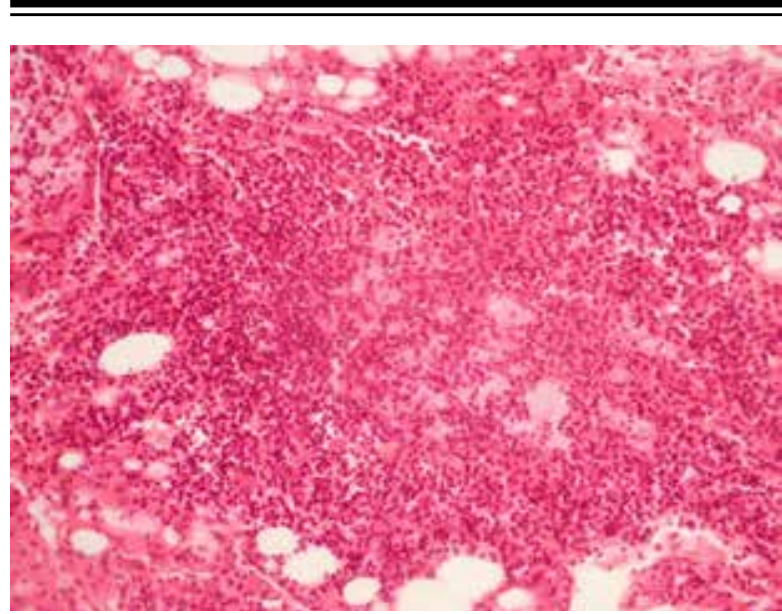

Рис. 2. Множественные эпителиоидные клетки с эозинофильной цитоплазмой и увеличенными ядрами, адипоциты и синусоидальные сосуды. Окраска - гематоксилин-эозин. Ув. $\times 100$.

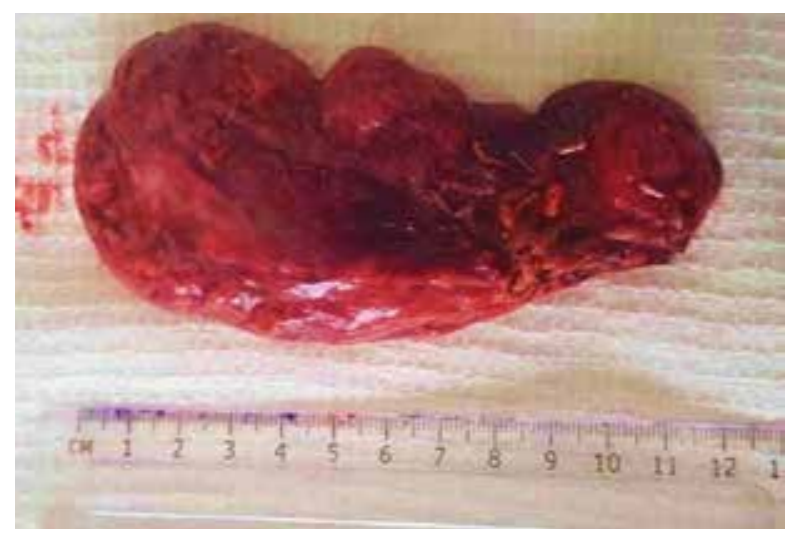

Рис. 4. Макропрепарат.

Макроскопически препарат представлял собой ограниченную, инкапсулированную массу размерами $82 \times 93 \times 105$ мм, поверхность разреза была однородной, светло-коричневого цвета, без участков некроза, с наличием очаговых кровоизлияний (рис. 4).

Гистологические препараты были направлены на иммуногистохимическое исследование.

Ниже приведены результаты иммуногистохимического исследования удаленного препарата. Микроскопически опухоль была представлена зрелой жировой тканью, кровеносными сосудами и гладкомышечными клетками, причем преобладали элементы гладких мышц (рис. 5).

Ядра жировых клеток были пикнотичны, расположены эксцентрично. Сосудистый компонент характеризовался как разветвленной сетью капилляров, так и извитыми толстостенными сосудами, напоминающими артерии. Однако в крупных сосудах отмечалось снижение экспрессии гладкомышеч-

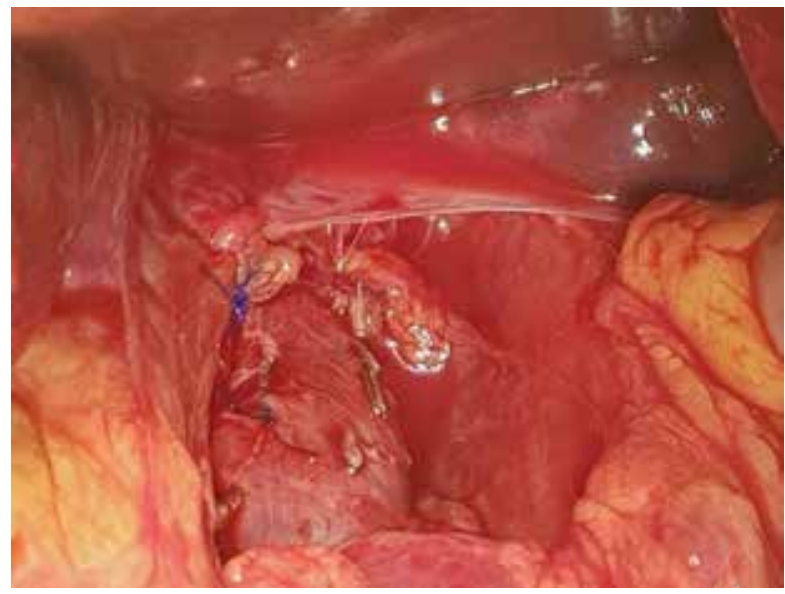

Рис. 3. Вид операционного поля после удаления опухоли Sg1 печени.

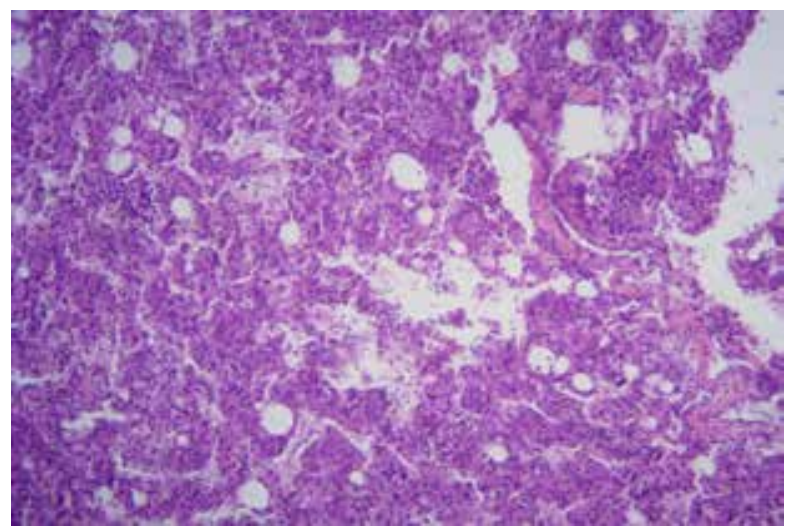

Рис. 5. Морфологическое строение опухоли печени: гладкомышечные структуры, сосуды, зрелая жировая ткань. Окраска - гематоксилин-эозин. Ув. $\times 100$.

ного актина и признаки фиброзирования. Гладкомышечные структуры образовывали вокруг сосудов своеобразные «муфты»; ядра гладкомышечных клеток были гиперхромные, умеренно полиморфные, фигуры митозов выявлены не были.

Кроме гладкомышечного актина для иммунофенотипа ангиомиолипомы также характерна экспрессия меланоцитарных маркеров НMB-45 и Melan A (рис. 6).

Иммуногистохимические исследования с маркером пролиферации Ki67 и мутантным онкомаркером Р53 были негативными, что, наряду с неэпителиоидным характером данного образования, позволило сделать вывод о его доброкачественном характере и подтвердить диагноз: ангиомиолипома печени.

Послеоперационный период протекал гладко, без осложнений на фоне консервативной терапии, перевязок. Раны зажили первичным натяжением, швы сняты. Пациентка выписана на 12-е послеоперационные сутки. 


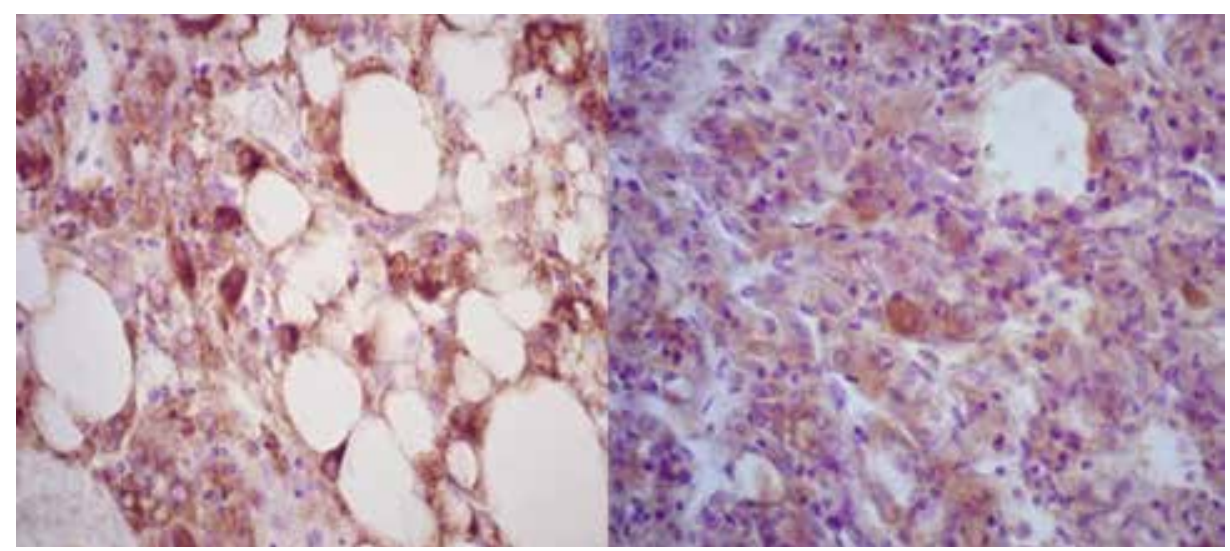

Рис. 6. Экспрессия меланоцитарных маркеров клетками опухоли печени. Реакция с МКАT к Melanosome НMB-45 (слева) и Melan A (A103) (справа). Ув. ×400.

\section{Обсуждение}

Впервые АМЛ была описана в 1976 году KG. Ishak [10] и в данный момент отнесена к семейству опухолей, возникающих из периваскулярных эпителиоидных клеток (PEComas), которые были классифицированы F. Bonetti в 1992 году [11, 12, 13].

Ангиомиолипома печени очень часто (более чем в 50\% случаев) неправильно диагностируется как гепатоцеллюлярная карцинома (ГЦК) из-за высокого сходства в КТ-картине. Дифференциальная диагностика между АМЛ и ГЦК очень важна, так как хирургическая тактика и прогноз радикально отличаются при множественном характере образований.

Случаи множественных ангиомиолипом печени и легких при отсутствии их в почках носят единичный характер в мировой литературе. Характер образований позволяет считать, что это множественный доброкачественный процесс, а не первичное образование с метастазами в печень и легкие. Тем не менее, в литературе описаны случаи злокачественных АМЛ печени, которые характеризуются большим размером опухоли, инвазивным ростом, гистологически наличием клеточной атипии и высокой активностью пролиферации (индекс Кi67 выше 30\%), что не всегда выявляется в дооперационном периоде при КТ ОБП и биопсии. Кроме того, злокачественная АМЛ проявляет способность к метастазированию и рецидивированию после радикального ее удаления, что влияет на показатели смертности [14, 15]. В описанном случае отсутствие инвазивного роста, атипичной митотической активности при гистологическом исследовании и негативные результаты с маркером пролиферации Кi67 и мутантным онкомаркером Р53 при иммуногистохимическом исследовании позволяют сделать вывод о доброкачественном характере АМЛ.

\section{Заключение}

Ангиомиолипома - редкая доброкачественная мезенхимальная опухоль печени с возможностью малигнизации. В статье представлен редкий клинический случай хирургического лечения пациента с мультифокальной ангиомиолипомой печени и легких. В связи со значительной схожестью ангиомиолипомы печени и гепатоцеллюлярной карциномы на спиральной компьютерной томографии и при микроскопии, дифференциальная диагностика имеет большое значение для соответствующей хирургической стратегии. Резекцию печени следует рассматривать как оптимальный вариант лечения крупной печеночной ангиомиолипомы. Небольшие очаги подлежат консервативному лечению и динамическому наблюдению. Оперативное вмешательство следует проводить в специализированных центрах с большим опытом выполнения резекций печени.

\section{Финансирование}

Работа выполнялась в соответствии с планом научных исследований Института общей и неотложной хирургии им. В.Т. Зайцева НАMНУ.

\section{Конфликт интересов}

Авторы заявляют, что конфликт интересов отсутствует.

\section{Этические аспекты Одобрение комитета по этике}

Одобрено комитетом по этике Института общей и неотложной хирургии им. В.Т. Зайцева НАМНУ (протокол №9 от 10.11.2018). 


\section{Согласие пациента}

Пациент дал согласие на публикацию сообщения и размещение в интернете информации о характере его заболевания, проведенном лечении и его результатах с научной и образовательной целями.

\section{ЛИТЕРАТУРА}

1. Fukuda Y, Omiya H, Takami K, Mori K, Kodama Y, Mano M, Nomura Y, Akiba J, Yano H, Nakashima O, Ogawara M, Mita E, Nakamori S, Sekimoto M. Malignant hepatic epithelioid angiomyolipoma with recurrence in the lung 7 years after hepatectomy: a case report and literature review. Surg Case Rep. 2016 Dec;2:31. Published online 2016 Apr 2. doi: 10.1186/s40792-016-0158-1

2. Deng YF, Lin Q, Zhang SH, Ling YM, He JK, Chen XF. Malignant angiomyolipoma in the liver: a case report with pathological and molecular analysis. Pathol Res Pract. 2008;204(12):911-18. doi: 10.1016/j. prp.2008.06.007

3. Nguyen TT, Gorman B, Shields D, Goodman Z. Malignant hepatic angiomyolipoma: report of a case and review of literature. Am J Surg Pathol. 2008 May;32(5):793-98. doi: 10.1097/PAS.0b013e3181607349 4. Wang WT, Li ZQ, Zhang GH, Guo Y, Teng MJ. Liver transplantation for recurrent posthepatectomy malignant hepatic angiomyolipoma: a case report. World J Gastroenterol. 2015 Mar 28;21(12):3755-58. Published online 2015 Mar 28. doi: 10.3748/wjg.v21. i12.3755

5. Saito M, Tsukamoto T, Takahashi T, Sai K, Fujii $\mathrm{H}$, Nagashima K. Multifocal angiomyolipoma affecting the liver and lung without tuberous sclerosis. J Clin Pathol. 2004 Feb;57(2):221-24. doi: 10.1136/ jcp.2003.10330

6. Shi H, Cao D, Wei L, Sun L, Guo A. Inflammatory angiomyolipomas of the liver: a clinicopathologic and immunohistochemical analysis of 5 cases. Ann Diagn Pathol. 2010 Aug;14(4):240-46. doi: 10.1016/j. anndiagpath.2010.03.002

7. Makhlouf HR, Ishak KG, Shekar R, Sesterhenn IA, Young DY, Fanburg-Smith JC. Melanoma markers in angiomyolipoma of the liver and kidney: a comparative study. Arch Pathol Lab Med. 2002 Jan;126(1):49-55. doi: 10.1043/0003-9985(2002)126<0049:MMIAOT>2.0. $\mathrm{CO} ; 2$

8. Zhou Y, Chen F, Jiang W, Meng Q, Wang F. Hepatic epithelioid angiomyolipoma with an unusual pathologic appearance: expanding the morphologic spectrum. Int J Clin Exp Pathol. 2014 Aug 15;7(9):636469. eCollection 2014. https://www.ncbi.nlm.nih.gov/ pmc/articles/PMC4203263/

9. Talati H, Radhi J, Popovich S, Marcaccio M. Hepatic epithelioid angiomyolipoma: case series. Gastroenterology Res. 2010 Dec;3(6):293-95. Published online 2010 Nov 20. doi: $10.4021 / \mathrm{gr} 273 \mathrm{w}$

10. Ishak KG. Mesenchymal tumors of the liver. In: Okuda K, Peters RL, editors. Hepatocellular carcinoma. New York: John Wiley and Sons; 1976. p. 247307.

11. Bonetti F, Pea M, Martignoni G, Zamboni G. PEC and sugar. Am J Surg Pathol. 1992 Mar;16(3):3078. https://journals.lww.com/ajsp/citation/1992/03000/ pec_and_sugar.13.aspx
12. Martignoni G, Pea M, Reghellin D, Zamboni G, Bonetti F. PEComas: the past, the present and the future. Virchows Arch. 2008 Feb;452(2):119-32. doi: 10.1007/s00428-007-0509-1

13. Folpe AL, Kwiatkowski DJ. Perivascular epithelioid cell neoplasms: pathology and pathogenesis. Hum Pathol. 2010 Jan;41(1):1-15. doi: 10.1016/j. humpath.2009.05.011

14. Yang CY, Ho MC, Jeng YM, Hu RH, Wu YM, Lee $\mathrm{PH}$. Management of hepatic angiomyolipoma. $J$ Gastrointest Surg. 2007 Apr;11(4):452-57. doi: 10.1007/ s11605-006-0037-3

15. Ding GH, Liu Y, Wu MC, Yang GS, Yang JM, Cong WM. Diagnosis and treatment of hepatic angiomyolipoma. J Surg Oncol. 2011 Jun;103(8):807-12. doi: $10.1002 /$ jso. 21814

\section{REFERENCES}

1. Fukuda Y, Omiya H, Takami K, Mori K, Kodama Y, Mano M, Nomura Y, Akiba J, Yano H, Nakashima O, Ogawara M, Mita E, Nakamori S, Sekimoto M. Malignant hepatic epithelioid angiomyolipoma with recurrence in the lung 7 years after hepatectomy: a case report and literature review. Surg Case Rep. 2016 Dec;2:31. Published online 2016 Apr 2. doi: 10.1186/s40792-016-0158-1

2. Deng YF, Lin Q, Zhang SH, Ling YM, He JK, Chen XF. Malignant angiomyolipoma in the liver: a case report with pathological and molecular analysis. Pathol Res Pract. 2008;204(12):911-18. doi: 10.1016/j. prp.2008.06.007

3. Nguyen TT, Gorman B, Shields D, Goodman Z. Malignant hepatic angiomyolipoma: report of a case and review of literature. Am J Surg Pathol. 2008 May;32(5):793-98. doi: 10.1097/ PAS.0b013e3181607349

4. Wang WT, Li ZQ, Zhang GH, Guo Y, Teng MJ. Liver transplantation for recurrent posthepatectomy malignant hepatic angiomyolipoma: a case report. World J Gastroenterol. 2015 Mar 28;21(12):3755-58. Published online 2015 Mar 28. doi: 10.3748/wjg.v21. i12.3755

5. Saito M, Tsukamoto T, Takahashi T, Sai K, Fujii H, Nagashima K. Multifocal angiomyolipoma affecting the liver and lung without tuberous sclerosis. J Clin Pathol. 2004 Feb;57(2):221-24. doi: 10.1136/ jcp.2003.10330

6. Shi H, Cao D, Wei L, Sun L, Guo A. Inflammatory angiomyolipomas of the liver: a clinicopathologic and immunohistochemical analysis of 5 cases. Ann Diagn Pathol. 2010 Aug;14(4):240-46. doi: 10.1016/j. anndiagpath.2010.03.002

7. Makhlouf HR, Ishak KG, Shekar R, Sesterhenn IA, Young DY, Fanburg-Smith JC. Melanoma markers in angiomyolipoma of the liver and kidney: a comparative study. Arch Pathol Lab Med. 2002 Jan;126(1):49-55. doi: 10.1043/0003-9985(2002)126<0049:MMIAOT $>2.0$. $\mathrm{CO} ; 2$

8. Zhou Y, Chen F, Jiang W, Meng Q, Wang F. Hepatic epithelioid angiomyolipoma with an unusual pathologic appearance: expanding the morphologic spectrum. Int J Clin Exp Pathol. 2014 Aug 15;7(9):636469. eCollection 2014. https://www.ncbi.nlm.nih.gov/ pmc/articles/PMC4203263/

9. Talati H, Radhi J, Popovich S, Marcaccio M. Hepatic epithelioid angiomyolipoma: case series. Gastroenterology Res. 2010 Dec;3(6):293-95. Published online 
2010 Nov 20. doi: $10.4021 / \mathrm{gr} 273 \mathrm{w}$

10. Ishak KG. Mesenchymal tumors of the liver. In Okuda K, Peters RL, editors. Hepatocellular carcinoma. New York: John Wiley and Sons; 1976. p. 247-307. 11. Bonetti F, Pea M, Martignoni G, Zamboni G. PEC and sugar. Am J Surg Pathol. 1992 Mar;16(3):3078. https://journals.lww.com/ajsp/citation/1992/03000/ pec_and_sugar.13.aspx

12. Martignoni G, Pea M, Reghellin D, Zamboni G, Bonetti F. PEComas: the past, the present and the future. Virchows Arch. 2008 Feb;452(2):119-32. doi: 10.1007/s00428-007-0509-1

\section{Адрес для корреспонденции}

61103, Украина,

г. Харьков, въезд Балакирева, д. 1,

Институт общей и неотложной

хирургии им. В.Т. Зайцева НАМНУ,

отделение хирургии печени

и желчевыводящих протоков,

тел. моб.: +38 0506 15-45-55,

e-mail: dr.chernyayev@gmail.com,

Черняев Никита Святославович

\section{Сведения об авторах}

Бойко Валерий Владимирович, д.м.н., профессор, член-корреспондент НАМН Украины, директор Института общей и неотложной хирургии им. В.Т. Зайцева НАМН Украины, г. Харьков, Украина.

https://orcid.org/0000-0003-4771-9699

Смачило Ростислав Михайлович, д.м.н., профессор, заведующий отделением хирургии печени и желчевыводящих путей, Институт общей и неотложной хирургии им. В.Т. Зайцева НАМН Украины, г. Харьков, Украина.

https://orcid.org/0000-0003-1237-0255

Черняев Никита Святославович, к.м.н., ассистент кафедры хирургии № 1, Харьковский национальный медицинский университет, г. Харьков, Украина.

https://orcid.org/0000-0002-6543-311X

Яковцова Ирина Ивановна, д.м.н., профессор, заведующий кафедрой патологической анатомии Харьковская медицинская академия последипломного образования, г. Харьков, Украина.

https://orcid.org/0000-0002-1027-9215

Данилюк Светлана Владимировна, к.м.н., доцент кафедры патологической анатомии Харьковская медицинская академия последипломного образования, г. Харьков, Украина.

https://orcid.org/0000-0002-9971-0410

Тищенко Александр Михайлович, д.м.н., профессор отделения хирургии печени и желчевыводящих путей, Институт общей и неотложной хирургии им. B.Т. Зайцева НАМН Украины, г. Харьков, Украина. https://orcid.org/0000-0002-9151-778X

Веселый Андрей Леонидович, врач-рентгенолог кабинета компьютерной томографии, Институт общей и неотложной хирургии им. В.Т. Зайцева НАМН Украины, г. Харьков, Украина. https://orcid.org/0000-0002-5350-4259

\section{Информация о статье}

Получена 29 ноября 2018 года.

Принята в печать 27 мая 2019 г.

Доступна на сайте 1 июля 2019 г.
13. Folpe AL, Kwiatkowski DJ. Perivascular epithelioid cell neoplasms: pathology and pathogenesis. Hum Pathol. 2010 Jan;41(1):1-15. doi: 10.1016/j. humpath.2009.05.011

14. Yang CY, Ho MC, Jeng YM, Hu RH, Wu YM, Lee PH. Management of hepatic angiomyolipoma. $J$ Gastrointest Surg. 2007 Apr;11(4):452-57. doi: 10.1007/ s11605-006-0037-3

15. Ding GH, Liu Y, Wu MC, Yang GS, Yang JM, Cong WM. Diagnosis and treatment of hepatic angiomyolipoma. J Surg Oncol. 2011 Jun;103(8):807-12. doi: $10.1002 /$ jso. 21814

\section{Address for correspondence}

61103, Ukraine,

Kharkiv, Balakirev Entry, 1,

V.T. Zaytsev Institute of General and Urgent Surgery

Of the National Academy of Medical

Sciences of Ukraine,

Surgery Department of the Liver and Bile Ducts.

Tel. mobile: +380506 15-45-55,

e-mail: dr.chernyayev@gmail.com,

Mykyta S. Chernyayev

\section{Information about the authors}

Boyko Valeriy V., MD, Professor, Corresponding Member of the National Academy of Medical Sciences of Ukraine, Director of V.T. Zaytsev Institute of General and Urgent Surgery of the National Academy of Medical Sciences of Ukraine, Kharkiv, Ukraine.

https://orcid.org/0000-0003-4771-9699

Smachylo Rostyslav M., MD, Professor, Head of the Surgery Department of the Liver and Bile Ducts, V.T. Zaytsev Institute of General and Urgent Surgery of the National Academy of Medical Sciences of Ukraine, Kharkiv, Ukraine.

https://orcid.org/0000-0003-1237-0255

Chernyayev Mykyta S, PhD, Assistant of the Surgery Department № 1, Kharkiv National Medical University, Kharkiv, Ukraine.

https://orcid.org/0000-0002-6543-311X

Yakovtsova Iryna I., MD, Professor, Head of the Pathologic Anatomy Department, Kharkiv Medical Academy of Post-graduate Education, Kharkiv, Ukraine. https://orcid.org/0000-0002-1027-9215

Danyliuk Svitlana V., PhD, Associate Professor of the Pathologic Anatomy Department, Kharkiv Medical Academy of Post-graduate Education, Kharkiv, Ukraine. https://orcid.org/0000-0002-9971-0410

Tishchenko Olexander M., MD, Professor of the Surgery Department of the Liver and Bile Ducts, V.T. Zaytsev Institute of General and Urgent Surgery of the National Academy of Medical Sciences of Ukraine, Kharkiv, Ukraine.

https://orcid.org/0000-0002-9151-778X

Veselyi Andrii L., Radiologist of the Computed Tomography Cabinet, V.T. Zaytsev Institute of General and Urgent Surgery of the National Academy of Medical Sciences of Ukraine, Kharkiv, Ukraine.

https://orcid.org/0000-0002-5350-4259

\section{Article history}

Arrived: 29 November 2018

Accepted for publication: 27 May 2019

Available online: 1 July 2019 